УДК 622.83:[528.2:629.78]

ISSN 1609-0691

DOI: https://doi.org/10.17308/geology.2020.4/3122

Поступила в редакцию: 20.06.2020

Принята к публикации: 01.12.2020

Опубликована онлайн: 18.12 .2020

\title{
Экспериментальные исследования и визуализация современных тектонических движений восточной части Воронежского кристаллического массива
}

\author{
C)2020 А. А. Панжин ${ }^{凶}$ \\ Институт горного дела Уральского отделения РАН, \\ ул. Мамина-Сибиряка, 58, 620075, Екатеринбург, Российская Федерация
}

\begin{abstract}
Аннотация
Введение: Рассматриваются методика и результаты экспериментальных исследований современных тектонических движений Воронежского кристаллического массива (ВКМ) с использованием данных деформационного мониторинга, и связанные с ними вопросы моделирования природных, техногенных, и экологических систем.

Методика: Обозначены основные положения методики мониторинга и визуализации современных тектонических движений в виде векторного поля по результатам цикловых геодезических измерений. Результаты и обсуждение: Показано, что в качестве ключевого источника информации о тектонических движениях, проводимых на больших пространственно-временных базах, целесообразно использовать не абсолютные значения величин векторов сдвижений наблюдательных пунктов, а их скорости, приведенные к годовому циклу.

Заключение: На основании экспериментальных данных установлен вихревой характер современных тектонических движений как индикатор активных тектонических нарушений.

Ключевые слова: тектонические движения, деформации, мониторинг, визуализация, геологические структуры, векторное поле, дивергенция

Источник финансирования: Работа выполнена в рамках государственного задания № 075-00581-1900. Тема № 0328-2019-0005.

Для циттирования: Панжин А. А. Экспериментальные исследования и визуализация современных тектонических движений восточной части Воронежского кристаллического массива // Вестник Воронежского государственного университета. Серия: Геология. 2020. №4. С. 4-11. DOI: doi.org/10.17308/geology.2020.4/3122.
\end{abstract}

\section{Введение}

Вопрос возможности исследования современных тектонических движений с использованием данных деформационного мониторинга, проводимых как в режиме дискретных наблюдений на пунктах геодезической сети, так и с использованием денных постоянно действующих станций (GNSS), неоднократно рассмат- ривался в связи с исследованием современной геодинамики Уральского региона, вызванной как естественными, так и техногенными факторами $[1,2]$.

Определение цикловых пространственных координат наблюдательных пунктов и их изменений во времени осуществляется, в зависимости от размеров сетей, методами Precise Point Positioning (PPP) [3] для больших

Контент доступен под лицензией Creative Commons Attribution 4.0 License.

\footnotetext{
Панжин Андрей Алексеевич, e-mail: panzhin@igduran.ru
} 
сетей, и классическими методами Double Difference (DD) для локальных сетей. Также методы PPP и DD могут использоваться в комбинации, при этом методами DD производится расчет и уравнивание геодезической сети, а методом РРP - привязка локальных и региональных геодезических построений к международной сети IGS.

Важным аспектом при исследовании и визуализации современных тектонических движений является геоинформационное моделирование природных, техногенных, и экологических систем, которое позволяет выявить многие необходимые для их анализа свойства и характеристики, в том числе скрытые закономерности их пространственно-временного распределения. При этом многое зависит от исследования, адекватного объекту и его компонентам: идентификации и визуализации как количественной, так и качественной пространственно-временной информации [4]. Также визуализация тектонических движений по результатам цикловых геодезических измерений позволяет более обоснованно выделять активные геологические структуры, блоки, тектонические разломы, что необходимо для прогнозирования мест возможных сейсмических событий и принятия профилактических мер для обеспечения безопасности населения, промышленных объектов и др.

\section{Методика эксперимента}

Исследования современных тектонических движений в представленной работе осуществлялись в во- сточной части Воронежского кристаллического массива (ВКМ) с использованием данных деформационного мониторинга, проводимых постоянно действующими станциями GNSS на территории региона (рис. 1). Чтобы иметь возможность проследить и оценить вероятную связь современных геодинамических движений с тектоническим строением региона, на рис. 1 совмещено размещение пунктов GNSS с основными разломами региона, тектонические нарушения более низких рангов, выделяемые различными исследователями [5], не показаны.

Также на исследуемом участке зафиксирован эпицентр сейсмического события, отнесенного к природным землетрясениям. Магнитуда землетрясения, произошедшего 31.03.2000, составила 3.9, глубина очага 10 километров. По последствиям землетрясения такой магнитуды не представляют серьезной опасности, но они, являясь динамической реализацией предшествующих деформационных процессов, в определенной мере характеризуют современную геодинамическую активность района.

Всего на исследуемой территории, на площади размерами 200х300 км расположено 18 пунктов GNSS, которые производят накопление данных, что позволяет выполнить их высокоточную геодезическую привязку к общемировой сети IGS в режиме цикловых мониторинговых измерений.

В цикловых измерениях определение абсолютных пространственных координат наблюдательных пунктов

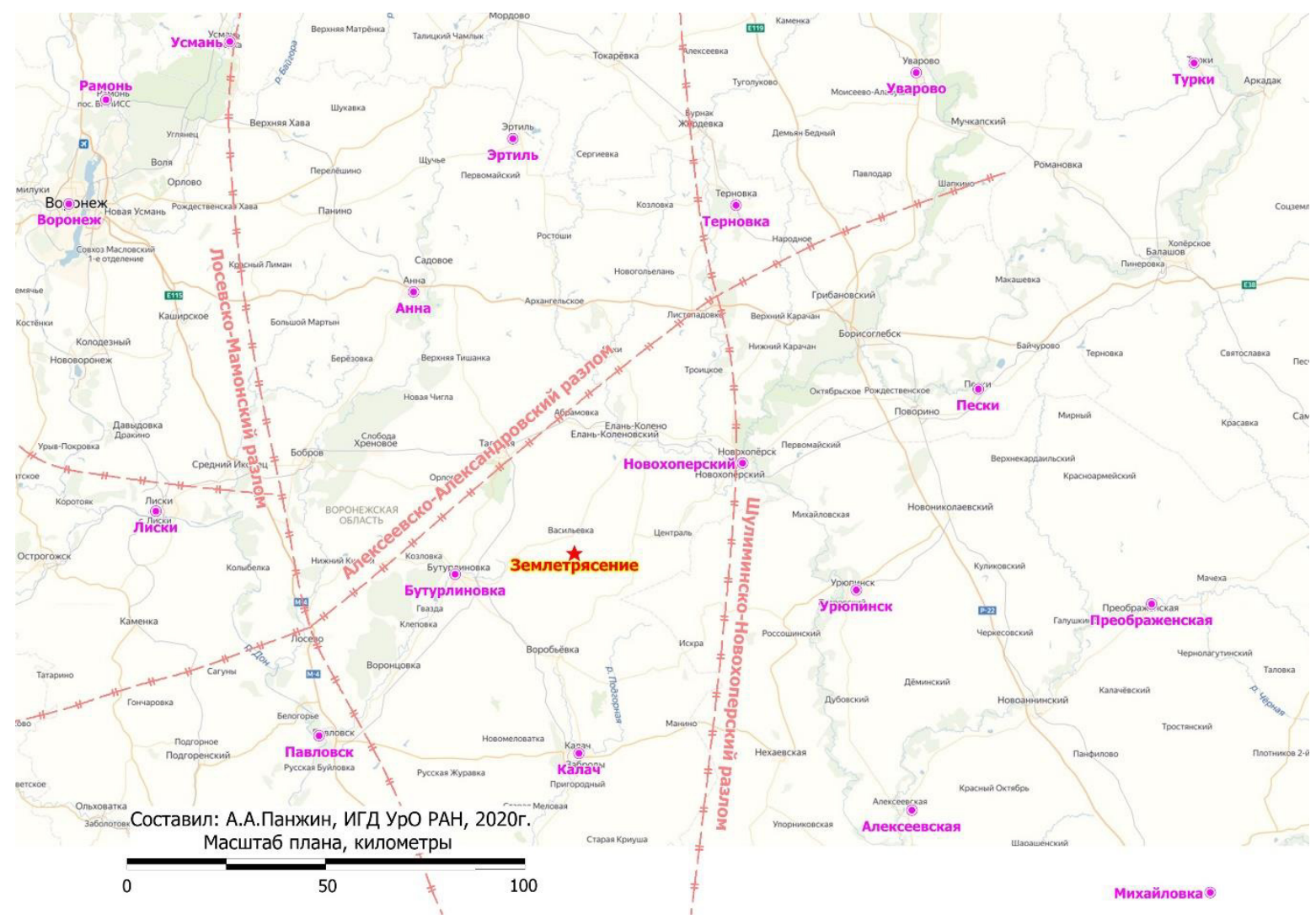

Рис. 1. Ситуационная карта расположения станций GNSS, по которым определялись современные геодинамические движения. [Fig. 1. Situational map of the location of GNSS stations, which were used to determine modern geodynamic movements.] 
осуществлялось периодически, четыре раза в год, для этого использовались накопленные станциями GNSS исходные файлы данных спутниковых навигационных приёмников в формате RINEX (Receiver Independent Exchange Format).

По каждой серии цикловых измерений проводилось 3-5 суточных наблюдений на каждом пункте GNSS, что позволяло определить их пространственные координаты с погрешностью $2 \delta$, на превышающей \pm 5 мм в горизонтальной и \pm 7 мм в вертикальной плоскости.

Камеральная обработка, с получением пространственных координат наблюдательных пунктов в Международной наземной опорной системе отсчета ITRF2014, производилась в пакетах программного обеспечения Bernese Software (методом Precise Point Positioning PPP) и Waypoint GrafNet (методом Double Difference DD) с определением пространственных координат пунктов по каждой суточной серии наблюдений с последующей статистической обработкой массива данных.

В работе [6] показано, в качестве ключевого источника информации о тектонических движениях по результатам мониторинговых измерений, проводимых на больших пространственно-временных базах, целесообразно использовать не абсолютные значения величин векторов сдвижений пунктов GNSS и реперов наблюдательных станций, а их скорости, приведенные к годовому циклу. Приведение скоростей к годовому циклу необходимо при проведении мониторинговых измерений по нерегулярным по времени цикловым измерениям для приведения результатов к общей пространственно-временной базе.

\section{Результаты исследований}

Годовые скорости векторов трендовых движений пунктов были определены на основе анализа изменений их пространственных приращений координат $\Delta \mathrm{X}$, $\Delta \mathrm{Y}, \Delta \mathrm{Z}$, происшедших в промежутках между повторными циклами измерений, и представлены на рис 2. Направления и величины векторов достаточно однородны, что отражает общее преимущественно восточное трендовое направление литосферной плиты, включающей ВКМ. На исследуемом участке, на фоне трендовой составляющей (7.8 мм/год по широте, 35.9 мм/год по долготе, 7.6 мм/год по высоте) также отмечается неоднородность скоростей тектонических движений в горизонтальной и вертикальной плоскостях.

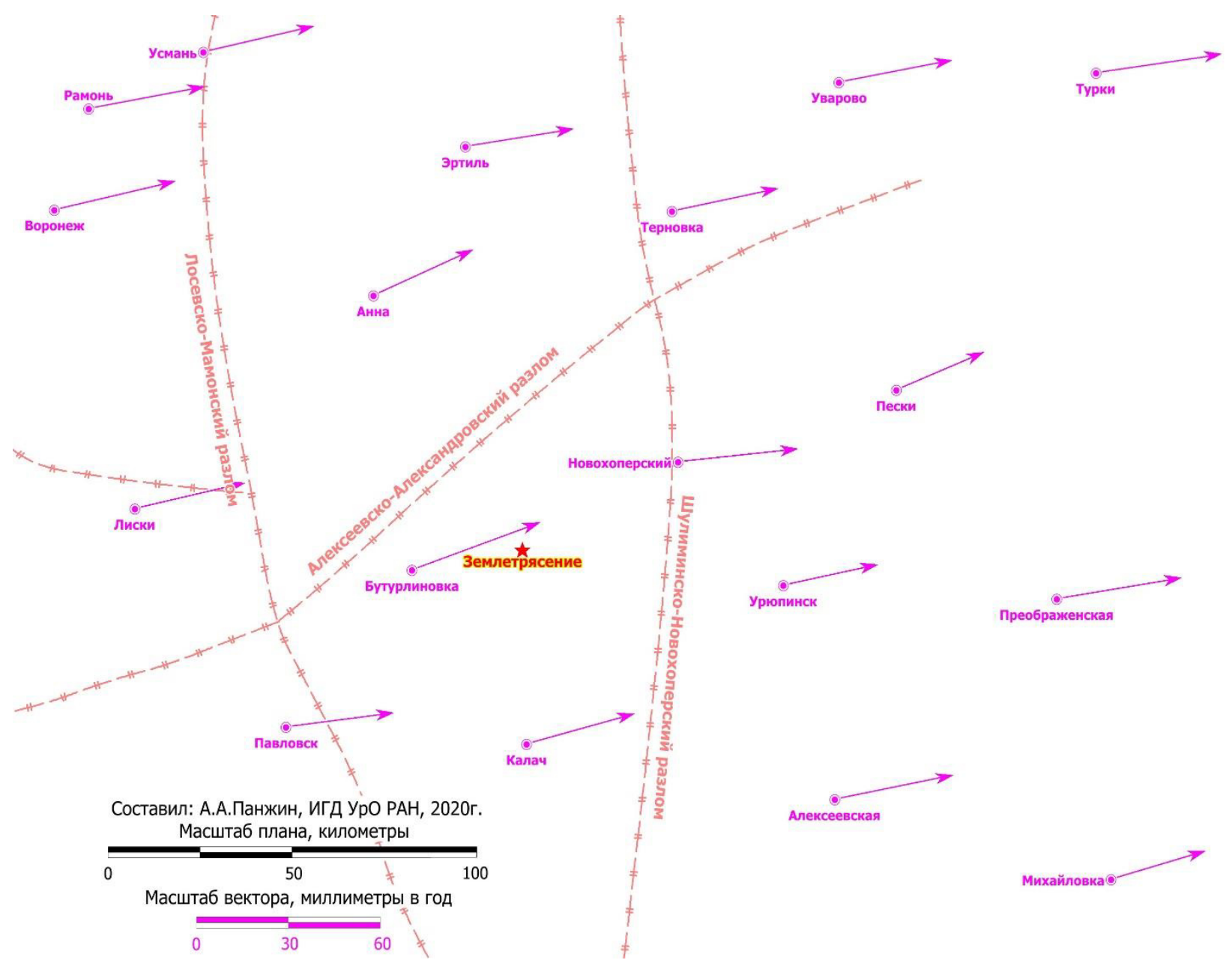

Рис. 2. Векторы трендовых горизонтальных геодинамических сдвижений.

[Fig. 2. Vectors of trending horizontal modern geodynamic movements.] 
В качестве ключевого источника информации о тектонических движениях по результатам мониторинговых измерений, проводимых на больших пространственно-временных базах, целесообразно использовать не абсолютные значения величин векторов сдвижений пунктов GNSS и реперов наблюдательных станций, а их скорости, приведенные к годовому циклу. Это необходимо при проведении мониторинговых измерений по нерегулярным по времени цикловым измерениям для приведения результатов к общей пространственно-временной базе.

Также анализ скоростей тектонических движений, а не их абсолютных значений, целесообразно использовать при исследовании процесса сдвижения при подземной и открытой разработках месторождений полезных ископаемых, поскольку в условиях действующего горнодобывающего предприятия возникает проблема утраты реперов наблюдательной станции. Это приводит к частичной ротации реперов вследствие восстановления уничтоженных и добавления новых в геодезические построения. При этом сопоставление абсолютных величин сдвижений и деформаций, полученных в различные периоды времени будет некорректным при использовании традиционных алгоритмов оценки и анализа деформационного состояния массива.
В результате, для каждой серии цикловых измерений определяются изменения пространственных приращений координат $\Delta \mathrm{X}, \Delta \mathrm{Y}, \Delta \mathrm{Z}$ пунктов, которые, для случая ВКМ, имеют преимущественно восточное направление за счет трендовой составляющей. При этом векторное поле деформаций визуализируется практически однородным, что не позволяет качественно идентифицировать пространственно-временную информацию о геодинамических движениях. Для числовой фильтрации трендовой составляющей применялся метод наименьших квадратов, с наложением условия математического минимума квадратов векторов сдвижений по каждой оси координат X, Y, Z.

$$
\sum \delta_{\mathrm{x}} \delta_{\mathrm{x}} \rightarrow \min ; \sum \delta_{\mathrm{y}} \delta_{\mathrm{y}} \rightarrow \min ; \sum \delta_{\mathrm{z}} \delta_{\mathrm{z}} \rightarrow \min .
$$

В результате определено и графически построено освобожденное от трендовой составляющей поле векторов тектонических движений исследуемой восточной части территории ВКМ, в котором визуально определяются вихревые сдвижения (рис. 3). Важность визуализации вихревых (ротационных) тектонических движений объясняется тем, что границы вихрей формируются по границам крупных подвижных структурных нарушений, что позволяет их идентифицировать активные тектонические структуры района [7, 8].

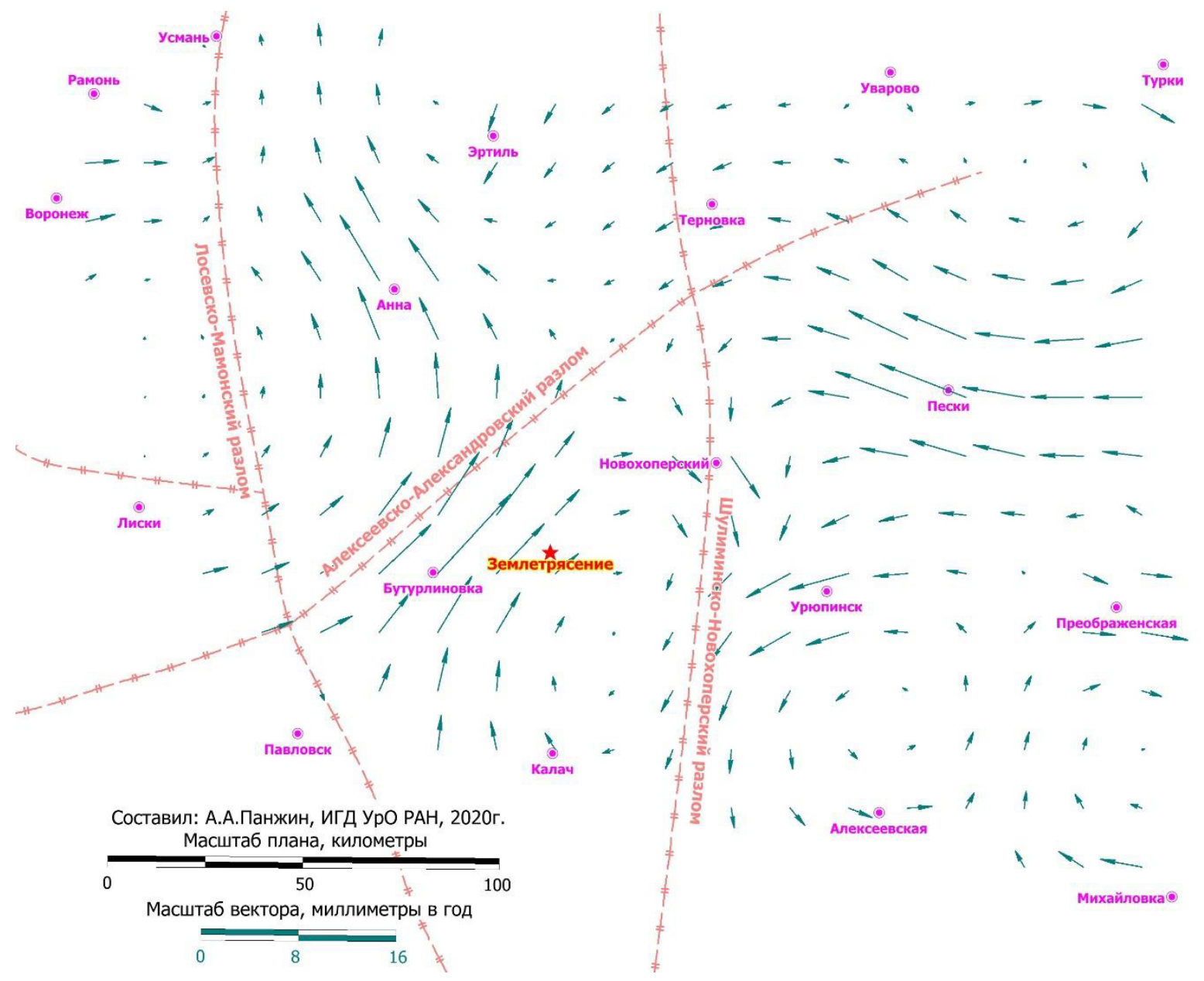

Рис. 3. Вихревые движения на исследуемой территории.

[Fig. 3. Vortex motions in the study area.] 


\section{Обсуждение результатов}

Полученные в результате измерений величины скоростей тектонических движений, с использованием математического аппарата механики сплошной среды преобразуются также в векторное и тензорное представление деформационного поля с выделением главных компонентов тензора деформаций (рис. 4). Это позволяет выделить и идентифицировать зоны с максимальными значениями главных деформаций как растяжения, так и сжатия, а также зон с повышенными значениями сдвиговых деформаций, приводящих к разрушениям объектов инфраструктуры.
Другой важной характеристикой векторного поля тектонических движений является дивергенция, которая характеризует степень сходимости или расходимости векторного потока, знание закономерностей распределения ее характеристик позволяет идентифицировать источники формирования и стока деформационных процессов, и определить их пространственное положение. Разработан и алгоритмически реализуется математический аппарат $[9,10]$, позволяющий определять дивергенцию по результатам исходных данных, представленных как в виде равномерной Крайгинг-модели, так и в виде данных, представленных в вершинах единичных элементов триангуляции Делоне.

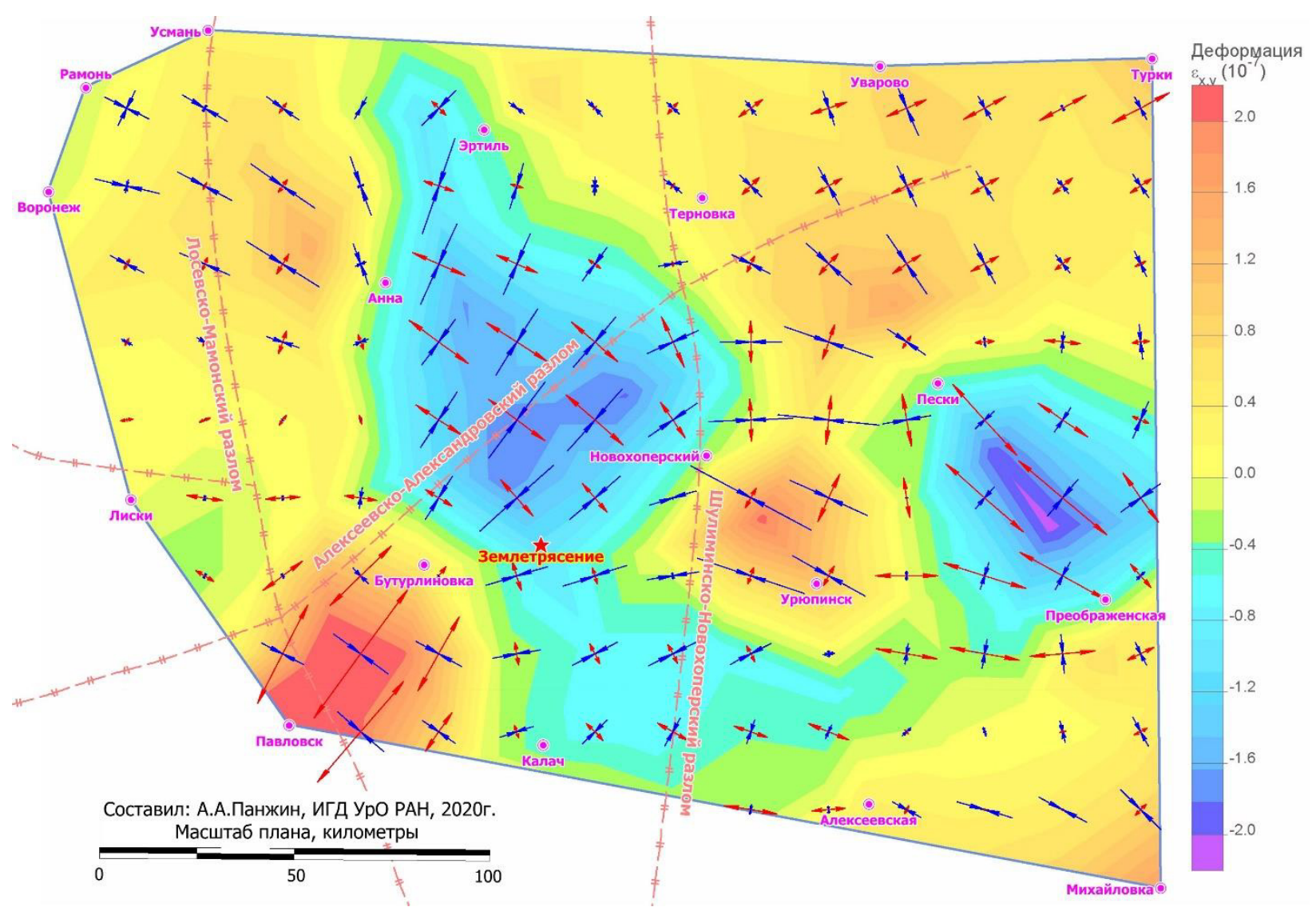

Рис. 4. Совмещенная схема поля тензоров горизонтальных главных деформаций (стрелки) и уровня сдвиговых деформаций (цвет, шкала справа).

[Fig. 4. Combined diagram of the field of tensors of main horizontal strains (arrows) and the level of shear strains (color scale on the right).]

\section{Заключение}

Таким образом, в статье обозначены основные положения методики мониторинга и визуализации современных тектонических движений в виде векторного поля по результатам цикловых геодезических измерений. На основании экспериментальных данных, полученных на ВКМ по результатам деформационного мониторинга по постоянно действующими на территории региона станциям GNSS, установлен вихревой характер современных тектонических движений как индикатор активных тектонических нарушений. Это позволяет на основании моделирования выделять активные геологические структуры, блоки, тектонические разло- мы за счет анализа поля сдвижений и деформаций, что необходимо для прогнозирования мест возможных сейсмических событий и обоснования профилактических мер по обеспечению безопасности населения, промышленных объектов и др. [11].

Другими вопросами визуализации тектонических движений по результатам мониторинговых измерений, требующими дальнейшей проработки, но не затронутыми на данном этапе, являются:

- Окончательная реализация в виде компьютерной программы разработанного математического аппарата оценки дивергенции векторных полей, что позволит определять дивергенцию по результатам исходных 
геодезических данных.

- Решение вопроса о возможности повышения оперативности получения результатов геодинамических исследований за счет использования в расчетах точных эфемерид разного класса (Rapid vs Final).

- Решение вопроса о выборе минимально возможного интервала между цикловыми сериями инструментальных измерений, при котором погрешности единичных измерений будут оказывать минимальное влияние на результат определения скоростей современных тектонических движений.

На основе экспериментальных данных определения скоростей современных геодинамических движений ВКМ в Международной земной системе координат ITRF2014 в настоящее время формируется «База данных современных геодинамических движений Воронежского кристаллического массива». В базе данных будет представлена информация о скоростях современных геодинамических движений, по которым возможно выявление областей высокоградиентных движений и концентрации деформаций ВКМ. База данных может использоваться для получения информации о геодинамической активности массива горных пород, предназначенного для расположения сложных и ответственных объектов промышленной инфраструктуры с целью обеспечения их устойчивости и безопасности. Информация, представленная в базе данных может быть использована для выявления и идентификации активных тектонических структур региона и геодинамического районирования ВКМ.

Благодарности. Автор выражает глубокую признательность коллективам компаний Эффективные технологии (www.eftgroup.ru), HIVE (www.hive.geosystems. aero), ГеоСтройИзыскания (www.gsi.ru) за предоставленные исходные данные и содействие при выполнении исследований.

Конфликт интересов: Автор декларирует отсутствие явных и потенциальных конфликтов интересов, связанных с публикацией настоящей статьи.

\section{ЛИТЕРАТУРА}

1. Уткин В. И., Белоусова А. А., Тягунов Д. С., Баландин Д. В. Исследование геодинамики Северного и Среднего Урала по данным GPS // Доклады Академии наук. 2010. Т. 431 № 2. C. 246-251.

2. Панжин А. А. Исследование геодинамических движений CORS для обоснования методики контроля процесса сдвижения на месторождениях Уральского региона // Вестник Магнитогорского государственного технического университета им. Г.И. Носова. 2015. № 1 (49). С. 22-26.

3. Zumberge J. F., Heflin M. B., Jefferson D. C., Watkins M. M., Webb, F.H. Precise point positioning for the efficient and robust analysis of GPS data from large networks // Journal of Geophysical Research: Solid Earth. 1997. T. 102. № B3. C. 5005-5017.

4. Мазуров Б. Т., Панжин А. А., Силаева А. А. Структурное моделирование полученных по геодезическим данным сдвижений путем визуализации // Геодезия и картография. 2016. № 3. C. 35-40. DOI: 10.22389/0016-7126-2016-909-3-25-40.

5. Трегуб А. И., Шевцов Д. Е. Разломы в фундаменте восточной части Воронежского кристаллического массива по морфометрическим данным // Вестник Воронежского государственного университета. Серия: Геология. 2020. №1. С. 30-38. DOI: https://doi.org/10.17308/geology. 2020.1/2511

6. Панжин А. А., Панжина Н. А. Оценка стабильности опорных пунктов как основы для геодинамического мониторинга // Известия высших учебных заведений. Горный журнал. 2019. № 6. C. 31-40. DOI: 10.21440/0536-1028-2019-6-31-40.

7. Мазуров Б. Т. Математическое моделирование при исследовании геодинамики. Новосибирск: СГУГиТ. 2019. 360 с.

8. Викулин А. В. Ротационные волны в блоковых вращающихся средах (на примере геологической среды) // Процессы в геосредах. 2016. № 7. С. 194-206.

9. Колмогоров В. Г., Мазуров Б. Т., Панжин А. А. Алгоритм оценки дивергенции векторных полей движений земной поверхности по геодезическим данным // Геодезия и картография. 2018. Т. 79. № 10. С. 46-53. DOI: 10.22389/0016-71262018-940-10-46-53.

10. Мазуров Б. Т., Мустафин М. Г., Панжин А. А. Метод оценки дивергенции векторных полей деформаций земной поверхности при разработке месторождений полезных ископаемых // Записки Горного института. 2019. Т. 238. № 4. С. 376-382. DOI: 10.31897/PMI.2019.4.376.

11. Сашурин А. Д., Панжин А. А. Современные проблемы и задачи геомеханики // Горный информащионно-аналитический бюллетень. 2020. № 3-1. С. 188-198. DOI: 10.25018/0236-1493-2020-31-0-188-198. 


\title{
GENERAL AND REGIONAL GEOLOGY
}

UDC 622.83:[528.2:629.78]

DOI: https://doi.org/10.17308/geology.2020.4/3122

ISSN 1609-0691

Received: 20.06.2020

Accepted: 01.12.2020

Published online: 18.12 .2020

\section{Experimental research and visualization of modern tectonic movements in the eastern part of the Voronezh crystalline massif}

\author{
CC2020 A. A. Panzhin ${ }^{凶}$ \\ Mining Institute of the Ural Branch of the Russian Academy of Sciences, \\ 58 Mamina-Sibiryaka st., Ekaterinburg 620075, Russian Federation
}

\begin{abstract}
Introduction: The article describes the methodology and the results of the experimental studies of modern tectonic movements of the Voronezh crystalline massif (VCM) using deformation monitoring data. It also discusses related issues of modelling natural, technogenic, and ecological systems.

Methodology: The study defined the main provisions of the methodology for monitoring and visualizing modern tectonic movements in the form of a vector field based on the results of cyclic geodetic measurements.

Results and discussion: It was shown that it is advisable to use the velocities of the displacement vectors of observation points as a key source of information on tectonic movements carried out on large space-time bases. It is not effective to use their absolute values.

Conclusions: The experimental data was used to establish the eddy nature of modern tectonic movements as an indicator of active tectonic disturbances.

Keywords: geodynamic movements, deformations, monitoring, visualization, geological structures, vector field, divergence

Funding: The study received financing within the framework of state order No 075-00581-19-00. Topic No. 0328-2019-0005.

For citation: Panzhin A. A. Experimental research and visualization of modern tectonic movements in the eastern part of the Voronezh crystalline massif. Vestnik Voronezhskogo gosudarstvennogo universiteta. Seriya: Geologiya $=$ Proceedings of Voronezh State University. Series: Geology. 2020, no. 4, pp. 4-11. DOI: doi.org/10.17308/geology.2020.4/3122.
\end{abstract}

Acknowledgments: The author thanks to the teams of the companies Effective Technologies (www.eftgroup.ru), HIVE (www.hive.geosystems.aero), GeoStroyIzyskaniya (www.gsi.ru) for providing the initial data and assistance in performing research.

Conflict of interests: The authors declare the absence of obvious and potential conflicts of interest related to the publication of this article

\section{REFERENCES}

1. Utkin V. I., Belousova A. A., Tjagunov D. S., Balandin D. V. Issledovanie geodinamiki Severnogo i Srednego Urala po dannym GPS [Research of geodynamics of the Northern and Middle Urals according to GPS data]. Doklady Akademii nauk $=$ Reports of the Academy of Sciences, 2010, vol. 431, no 2, pp. 246-251. (in Russ.)
2. Panzhin A. A. Issledovanie geodinamicheskih dvizhenij CORS dlja obosnovanija metodiki kontrolja processa sdvizhenija na mestorozhdenijah Ural'skogo regiona [Research of geodynamic movements CORS to substantiate the methodology for controlling the displacement process in the fields of the Ural region]. Vestnik Magnitogorskogo gosudarstvennogo tehnicheskogo universiteta im. G.I. No-

The content is available under Creative Commons Attribution 4.0 License.

\footnotetext{
$\bowtie$ Andrey A. Panzhin, e-mail: panzhin@igduran.ru
} 
sova $=$ Bulletin of the Magnitogorsk State Technical University. G.I. Nosova, 2015. no (49), pp. 22-26. (in Russ.) 3. Zumberge J. F., Heflin M. B., Jefferson D. C., Watkins M. M. Precise point positioning for the efficient and robust analysis of GPS data from large networks. Journal of Geophysical Research: Solid Earth, 1997, vol. 102, no B3, pp. 5005-5017.

4. Mazurov B. T., Panzhin A. A., Silaeva A. A. Strukturnoe modelirovanie poluchennyh po geodezicheskim dannym sdvizhenij putem vizualizacii [Structural modeling of displacements obtained from geodetic data by visualization]. Geodezija $i$ kartografija $=$ Geodesy and cartography, 2016, no 3, pp. 35-40. DOI: 10.22389/00167126-2016-909-3-25-40. (in Russ.)

5. Tregub A. I., Shevtsov D. E. Faults in the basement of the Eastern part of the Voronezh Crystalline Massif according to morphometric data. Vestnik Voronezhskogo gosudarstvennogo universiteta. Seriya: Geologiya $=$ Proceedings of Voronezh State University. Series: Geology, 2020, no. 1, pp. 30-38. DOI: https://doi.org/10.17308/geology.2020.1/2511. (in Russ.)

6. Panzhin A. A., Panzhina N. A. Ocenka stabil'nosti opornyh punktov kak osnovy dlja geodinamicheskogo monitoring [Evaluation of the stability of control points as a basis for geodynamic monitoring]. Izvestija vysshih uchebnyh zavedenij. Gornyj zhurnal = News of the Higher Institutions. Mining Journal, 2019, no 6, pp. 3140. DOI: 10.21440/0536-1028-2019-6-31-40. (in Russ.) 7. Mazurov B. T. Matematicheskoe modelirovanie pri issledovanii geodinamiki [Mathematical modeling in the study of geodynamics]. Novosibirsk: Siberian State University of Geosystems and Technologies, 2019. 360 p. (in Russ.)

8. Vikulin A. M. Rotacionnye volny v blokovyh vrashhajushhihsja sredah (na primere geologicheskoj sredy) [Rotational waves in block rotating media (on the example of the geological environment)]. Processy $v$ geosredah $=$ Processes in geospheres, 2016, no 7. pp. 194-206. (in Russ.)

9. Kolmogorov V. G., Mazurov B. T., Panzhin A. A. Algoritm ocenki divergencii vektornyh polej dvizhenij zemnoj poverhnosti po geodezicheskim dannym [Algorithm for estimating the divergence of vector fields of movements of the earth's surface from geodetic data]. Geodezija $i$ kartografija $=$ Geodesy and cartography, 2018, vol. 79, no 10, pp. 46-53. DOI: 10.22389/0016-7126-2018-94010-46-53. (in Russ.)

10. Mazurov B. T., Mustafin M. G., Panzhin A. A. Metod ocenki divergencii vektornyh polej deformacij zemnoj poverhnosti pri razrabotke mestorozhdenij poleznyh iskopaemyh [Method for assessing the divergence of vector deformation fields of the earth's surface during the development of mineral deposits]. Zapiski Gornogo Instituta $=$ Journal of Mining Institute, 2019, vol. 238, no 4, pp. 376382. DOI: 10.31897/PMI.2019.4.376. (in Russ.)

11. Sashurin A. D., Panzhin A. A. Sovremennye problemy i zadachi geomehaniki [Modern problems and tasks of geomechanics]. Gornyj informacionno-analiticheskij bjullet$e n^{\prime}=$ Mining informational and analytical bulletin, 2020, no 3-1, pp. 188-198. DOI: 10.25018/0236-1493-2020-310-188-198.
Панжин Андрей Алексеевич - к.т.н., ученый секретарь, Институт горного дела Уральского отделения РАН, Екатеринбург, Российская Федерация; E-mail: panzhin@igduran.ru; ORCID http://orcid.org/0000-0001-7766-2668

Автор прочитал и одобрил окончательный вариант рукописи.
Andrey A. Panzhin - PhD in Tech., science secretary, Institute of Mining of the Ural Branch of the RAS, Ekaterinburg, Russian Federation; E-mail: panzhin@igduran.ru; ORCID http://orcid.org/0000-0001-7766-2668

Author have read and approved the final manuscript. 